\title{
Book Review - Buchbesprechung - Livre nouveau
}

\section{Cudkowicz: The Human Bronchial Circulation in Health and Disease.}

Williams \& Wilkins, Baltimore 19(38.

Schon im letzten Jahrhundert wurde von den Pathologen, z. B. bei der Pulmonalstenose und bei Bronchiektasen, eine Erweiterung der Bronchial-arterien beschrieben. Bisher wurde der Bronchialkreislauf beim lebenden Patienten nur an wenigen Zentren systematisch untersucht. Die Monographie bringt einleitend knapp, aber übersichtlich die Anatomie und die Physiologie des Bronchialkreislaufes sowie die Methoden für die Messung der Bronchial-durchblutung. Beim Menschen haben sich die Indikatorverdünnungsmethoden am besten bewährt.

Normalerweise fliessen weniger als $2 \%$ des vom linken Ventrikel geförderten Blutvolumens durch den Bronchialkreislauf. Dieser Anted kann bei verschiedenen pathologischen Bedingungen um ein Mehrfaches gesteigert sein.

Die routinemässige Anwendung dieser Messmethoden stösst aber in der Klinik noch auf erhebliche Schwierigkeiten. Der Autor hat selbst viele Messun-gen durchgeführt, dokumentiert aber die Verhältnisse bei kongenitalen Vitien, Bronchiektasien, Tuberkulose, Tumoren, Emphysem und Lungenfibrosen mit angiographischen und histologischen Befunden, nicht aber mit in vivo durch-geführten Messungen der Bronchialdurchblutung. Zweifellos ist die Monographie geeignet, das Interesse für diesen in der Klinik vernachlässigten Aspekt der Hämodynamik des Lungenkreislaufes zu erhöhen.

A. Bühlmann, Zurich 